\title{
Are there sex differences in geographic knowledge and understanding?
}

\author{
ROBERT M. KITCHIN \\ Department of Geography, School of Geosciences, Queen's University Belfast, BT7 1NN \\ This paper was accepted for publication in March 1996
}

\begin{abstract}
Research by both geographers and psychologists suggests that there are differences in females' and males' everyday geographic and spatial knowledge. These differences in knowledge have been attributed to variances in biology and hormonal levels; differences in social status, culture and education; and differences in the ability to answer questions and think about geographic space. This paper examines these theories and explores the everyday geographic knowledge of females and males using both quantitative exercises and qualitative interviews. In contrast to most studies, only a few minor differences were found between females' and males' knowledge, their ability to answer the questions set and the strategies of spatial thought employed and it is suggested that any differences found between the sexes in other studies are due to socio-cultural factors reinforcing gender stereotypes.
\end{abstract}

KEY WORDS: sex differences, cognitive map knowledge, spatial ability.

I T IS WIDELY REPORTED, in both the geography (e.g. Matthews, 1986) and psychology (e.g. Herman and Siegel, 1978) literature, that males and females differ in both their local geographic knowledge and in the ability to remember, comprehend, manipulate and communicate spatial concepts such as relative positioning. Many researchers suggest that this difference in knowledge and spatial ability is present across the life-span (e.g. Kirasic et al., 1992) with differences starting to appear as young as four years old (Siegel and Schadler, 1977). For example, Lord (1941) found that males were more accurate than females in reconstructing an automobile journey and the locations passed. Herman and Siegel (1978) reported that boys were significantly better at reconstructing the layout of a large-scale town model at both second and fifth grade, although no difference was found at kindergarten level. Siegel and Schadler (1977) found that four and a half to six year-old boys were better at constructing a three-dimensional model of their classroom. Hart (1979) discovered boys' models of the area they lived in to be better organized both relationally and metrically. Bettis (1974) tested the geographic knowledge of 1700 fifth-grade children about the state they lived in, by asking the children to interpret graphs and maps, name places and identify features. Boys outperformed girls on 42 of the questions and were equal on three (Harris, 1981). Beatty and Troster (1987), using 1800 undergraduates, found that males consistently outperformed women on tests of geographical knowledge, but females learnt new maps just as rapidly as males. Matthews (1986) using 166, six to 11 year-old children performing three tests (sketch map and interpretation of a map and aerial photograph) found differences in the quantitative accretion of environmental knowledge and the qualitative externalization about a place. By 11 , boys' maps were more differentiated, broader in conception, more detailed and demonstrated greater mapping ability than the girls' maps. Kirasic et al., (1992) have shown a difference in the ability of elderly women to learn about novel environments from slides.

Females and males differing in their knowledge and their ability to process spatial information raises two important questions. First, in terms of geographic education and assessing a student's capability on tasks requiring spatial skills, are females being unfairly disadvantaged by taking the same tests as males? If so, do we need to implement changes to remedy the balance between the sexes? Self and Golledge (1994) suggest that this may be the case if we want to redress the ratio of females to males in scientific or technical careers where spatial abilities 
are thought to be important. Second, do differences in geographic knowledge, particularly at a local scale, indicate variances in patterns of spatial behaviour and use of the environment? If so, why do females and males interact with space differently? How do their patterns of spatial behaviour differ? How are these differences gender-enforced? In recent years, feminist geographers have reported differences in how women interact with, and come to know, the wider world and how their patterns of behaviour are structured within the bounds of gender. For example, Pain (1991) and Valentine (1990) argue that women's interaction with certain spaces is limited through fear of personal crime. This has led Rose (1993: 16) to state that:

The limits on women's everyday activities are structured by what society expects women to be and therefore do.

As such, the aims of this paper are two-fold. First, to examine theories which seek to explain why males and females might differ in their knowledge of the physical environment and their understanding of geographical concepts relating to spatial relationships and associations. Second, to compare females' and males' ability to complete a series of cognitive mapping tasks that require knowledge of the local environment and the ability to manipulate and communicate spatial information. Cognitive mapping refers to our knowledge of the everyday environment and how we acquire, store, recall and decode such knowledge (Downs and Stea, 1973).

Why might the sexes differ in their answers to geographic tasks? As suggested, theories which seek to explain differences in the ability to complete cognitive mapping tasks centre upon possible differences in spatial competence or possible differences in underlying knowledge or a combination of the two. Self et al., (1992) argue that there are three main theories which seek to explain why females' and males' spatial products (externalized representation of their knowledge) may differ (interestingly this same categorization can be used to classify theories which seek to explain how visually impaired and blind individuals' spatial ability and knowledge differ from sighted individuals' [see Kitchin and Jacobson, forthcoming]). Proponents of the deficiency theory suggest that the sexes differ because of variances in human physiology and hormonal levels. Females thus achieve lower scores on cognitive mapping tasks because of reduced spatial ability. Proponents of the difference theory contend that the sexes differ because of a range of socio-cultural factors, such as early childhood training and expectations, parental and institutional expectations, stereotyping and experience, and differences in the courses taken at school level. Females thus achieve lower scores on cognitive map- ping tasks because of differing and more constrained access to the environment, and less access to situations that develop spatial skills. Proponents of the inefficiency theory suggest that spatial and cognitive mapping ability and knowledge are essentially the same for both sexes but that measuring tasks favour male problem-solving strategies. Females thus achieve lower scores not because of spatial ability or geographic knowledge, but because of general problem-solving strategies. Each of these is discussed in turn.

Advocates of the deficiency theory hypothesize that the patterns of brain organization, variations in the oestrogen or androgen content of the human body and genetic factors all influence spatial ability and competence (McGee, 1979). Theories concerning the brain's control of spatial abilities fall into two categories: bilateral cerebral control and lateralization. In the former, it is hypothesized that males outperform females because both sides of the brain control spatial ability. The left hemisphere, which controls language development, becomes dominant in females at an earlier age leading to an unbalanced pattern of organization (Bowers and LaBarba, 1988). This is possibly the result of foetal sex hormones which determine the relative rates of functional maturation. These hormones mean that the left hemisphere matures early in females and the right hemisphere matures early in males (Harris, 1981). The latter theory of lateralization is more popular. Here it is hypothesized that spatial abilities are related to the increasing lateralization of the visuospatial processing of the right cerebral hemisphere. It is thought that males utilize their right hemisphere to a greater extent than females, thus leading to better spatial abilities (Bowers and LaBarba, 1988).

Evidence concerning the various influences of hormones upon spatial ability can be found in the physiology development literature. It is reported that males' superiority in spatial ability starts at puberty, peaks at about 18 years old, and then slowly declines. This superiority parallels the production patterns of androgen in the male body. McGee (1982) notes that high body androgenization seems to be associated with low spatial test scores amongst males and higher test scores amongst females. He suggests that androgen alone may not be responsible for spatial ability but rather the body's balance of oestrogen-androgen. Additional evidence comes from studies of hormonal and chromosomal deficiency conditions and other research concerning androgen and spatial ability (Gilmartin and Patton, 1984). McGee (1982) hypothesized that spatial abilities are inheritable and may be linked to an $\mathrm{X}$-linked recessive gene. Females inherit two $\mathrm{X}$ chromosomes $(\mathrm{XX})$ whereas males only one (XY). Witelson and Swallow (1988) note that individuals with Turner's syndrome, who are 
missing an $\mathrm{X}$ chromosome and have reduced levels of oestrogen and androgen, show lower than normal levels of spatial ability. In addition, females with congenital adrenal hyperplasia $(\mathrm{CAH})$, who have higher levels of adrenal androgen, performed better than normal females on tests of spatial ability.

Advocates of the difference theory propose that men and women's actual cognitive map knowledge differs. For example, Goodchild (1974) and Moore (1979) suggest that women have a more detailed image of their immediate surroundings, whilst men have more composite images of a larger area. Matthews (1986) argues that this is the direct result of socialization, stereotyping and upbringing. Indeed, Gilmartin and Patton (1984) suggest that males and females are 'programmed' by society to conform to accepted, traditional gender roles, leading to differing patterns of spatial behaviour. As a result, women experience less interaction with the environment and receive stereotypical social and media pressure to move away from activities that build spatial and cognitive mapping abilities.

For example, the territorial movements of females are often more controlled than males with strictly defined ranges. This is particularly pronounced at younger ages, the time of most learning potential. Hart (1979) found that boys had greater freedom to explore, whereas girls were encouraged to stay in the safe confines of the home and to participate in the routines of family life. Liben (1981) suggests that if girls are less encouraged to explore and have less opportunity to acquire spatial knowledge they will have a reduced ability to assimilate information in the future. This difference in learning through exploration does not change with adulthood because historical roles, such as motherhood and housewifery, are spatially constraining (Brown and Broadway, 1981). In addition, women's patterns of movement are affected by other social factors such as the fear of personal crime, leaving parts of the environment unvisited and unexplored (Valentine, 1990).

Further, it is widely acknowledged that women who work, on average, are employed nearer to the home and have shorter commuting times (Preston et al., 1993). Reasons forwarded to explain these differences in spatial behaviour suggest that it is a rational response to lower wages (Rutherford and Wekerle, 1988); that women's labour market is spatially segregated and determines commuting times (Hanson and Pratt, 1988); that women are less mobile than men and more reliant on public transport (Hanson and Hanson, 1980); and that women often have more domestic responsibilities and choose employment near to home to allow a successful balance between home and work (Madden and White, 1980). In addition, when travelling further afield women are more often the passive traveller in a vehicle and, therefore, do not have to consciously think about the spatial layout or the route traversed. Self et al., (1992) argue, however, that women do now have as much active environmental interaction as men, which consists of more complex, but not necessarily longer travel patterns. Women, they contend, learn the local environment in greater detail carrying out shopping, children-related and recreational tasks, whereas men learn a larger area through travelling to work and socializing, but in less detail.

Advocates of the inefficiency theory suggest that we need to be cautious when generalizing about male superiority in tasks that require spatial ability. They argue that rather than cognitive map knowledge differing, the strategies of spatial thought might differ between the sexes. This would lead to different results on tests requiring spatial ability even though cognitive map knowledge may be the same. Blough and Slavin (1987) have suggested that females and males do employ different problem-solving strategies. They hypothesize that early verbal precocity in girls initiates a tendency to apply verbal solutions to visual-spatial problems, whereas boys are more likely to apply mental-spatial approaches more successfully. Social-cultural stereotyping reinforces these styles. They conclude that women are slower at spatial tasks, with slower mental rotation and mental comparisons, and a bias towards accuracy. As a result, cognitive mapping exercises utilizing speed reaction tests are more likely to favour boys.

Miller and Santoni (1986) suggest that cognitive map differences may occur because men may employ the strategy of using more Euclidean properties in their spatial products, whereas women may utilize more topological properties of space. They argue that this may be why differences at a young age are rare, where boys and girls share topological views of space, but by adolescence are common, when boys have developed Euclidean views. Matthews (1986) has also noted this, concluding that although boys were capable of conveying a Euclidean sense of place by the age of eight, girls found such skills 'tantalizingly elusive'. Spencer and Weetman (1981) found the style of sketch maps being employed by females to be predominantly sequential (route based), rather than spatial (area based). As sequential maps develop into spatial maps this suggests a lack of spatial development (Appleyard, 1970). McGuinness and Sparks (1983) have, however, found contrary evidence. They found on a campus sketch map test that females, although knowing where some of the routes were located, did not include them on their maps unless specifically asked to. They argue that the female approach to organizing topographic space is to group things such as landmarks, whereas males establish a set of coordinates such as the road network which provide a geometrical framework for the location of buildings.

Miller and Santoni (1986) hypothesize that these 


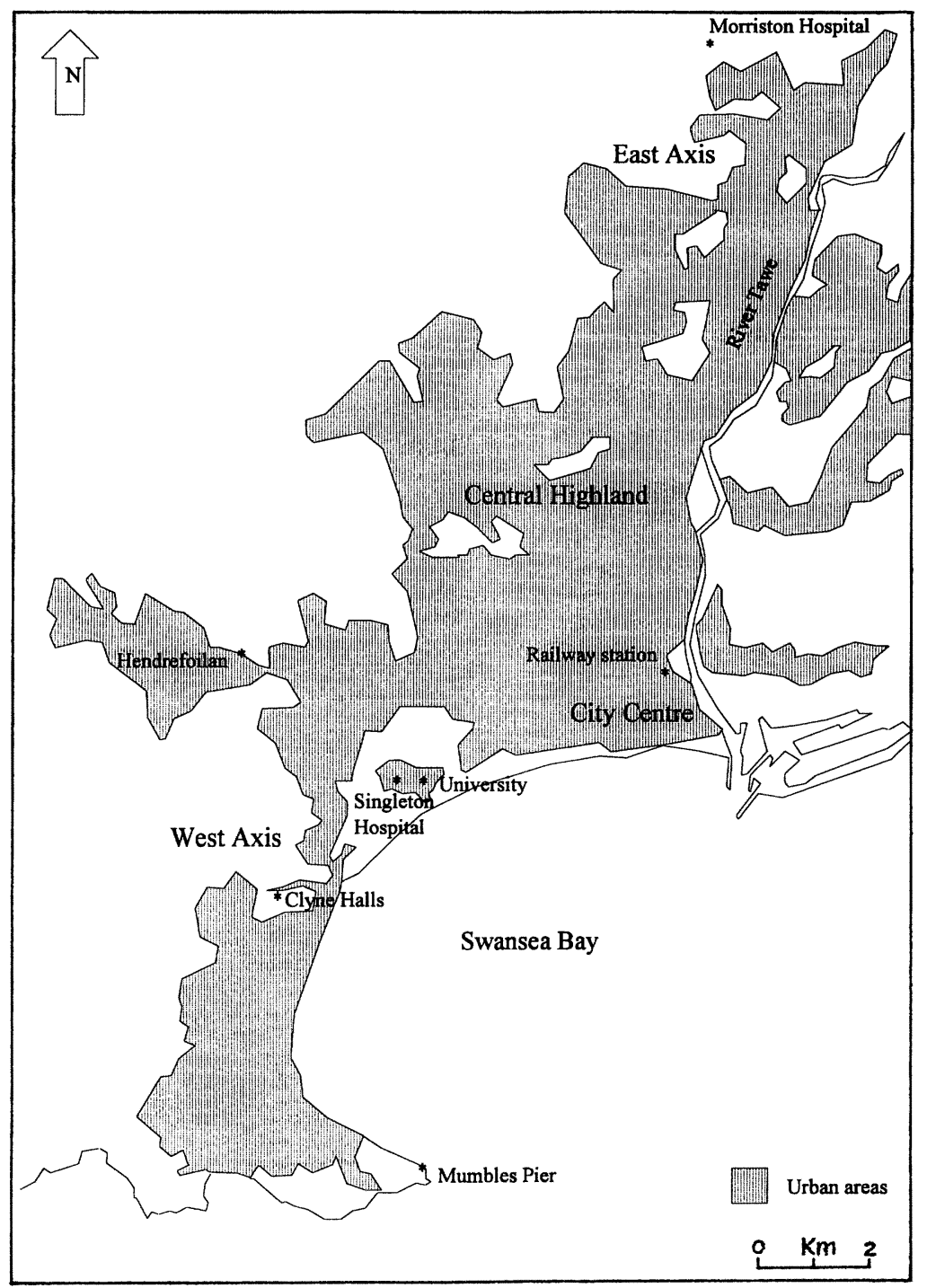

Fig. 1. Map of Srvansea

differences may be due to the informal differentiation of the school curriculum for males and females, leading to more sophisticated spatial skills for boys, although Spencer and Weetman (1981) found differences occurred regardless of geographical training. Liben (1981) notes that boys are encouraged to enrol on science and mathematics-based courses that develop spatial skills, whereas females take more language-based courses in which they perform better (Feingold, 1988). Self et al., (1992) and Golledge et al., (1993) have noted, however, that specific training can improve the results of both sexes, with the largest improvements in women.
It may be the case that girls suffer problems of metacognition, possessing less confidence in their ability to perform the required task. Metacognition concerns a respondent's awareness of their own performance or ability in a measurement exercise. Metacognition can be divided into the two main concepts of metamemory; 'knowing how to know' and 'knowing about knowing' (Allen, 1985). If we lack confidence, estimates could end up as guesses, and individuals who worry or avoid spatial behaviour and puzzles may have underdeveloped knowledge and skills (Liben, 1981). Poor metacognition may be enhanced by social stereotyping, and 
TABLE I

The number of respondents completing each test

\begin{tabular}{|c|c|c|c|c|c|c|c|}
\hline $\begin{array}{l}\text { Partial Graphic } \\
\text { test }\end{array}$ & $n^{0}$ & $\begin{array}{l}\text { Graphic } \\
\text { test }\end{array}$ & $n^{0}$ & $\begin{array}{l}\text { Uni-Multi } \\
\text { test }\end{array}$ & $n^{o}$ & $\begin{array}{l}\text { Recognition } \\
\text { test }\end{array}$ & $n^{o}$ \\
\hline Scr 1 & 19 & Sketch map 1 & 47 & $\begin{array}{l}\text { Projective } \\
\text { convergence }\end{array}$ & 41 & $\begin{array}{l}\text { Orientation } \\
\text { specification }\end{array}$ & 109 \\
\hline $\begin{array}{l}\text { Scr } 2 \\
\text { Scr } 3\end{array}$ & $\begin{array}{l}19 \\
14\end{array}$ & & & & & & \\
\hline $\begin{array}{l}\text { Scr } 4 \\
\text { Cz } 1\end{array}$ & $\begin{array}{l}33 \\
18\end{array}$ & Sketch map 2 & 31 & Metric MDS & 53 & & \\
\hline $\mathrm{Cz} 2$ & 26 & Sketch map 3 & 49 & Non-metric MDS & 38 & & \\
\hline
\end{tabular}

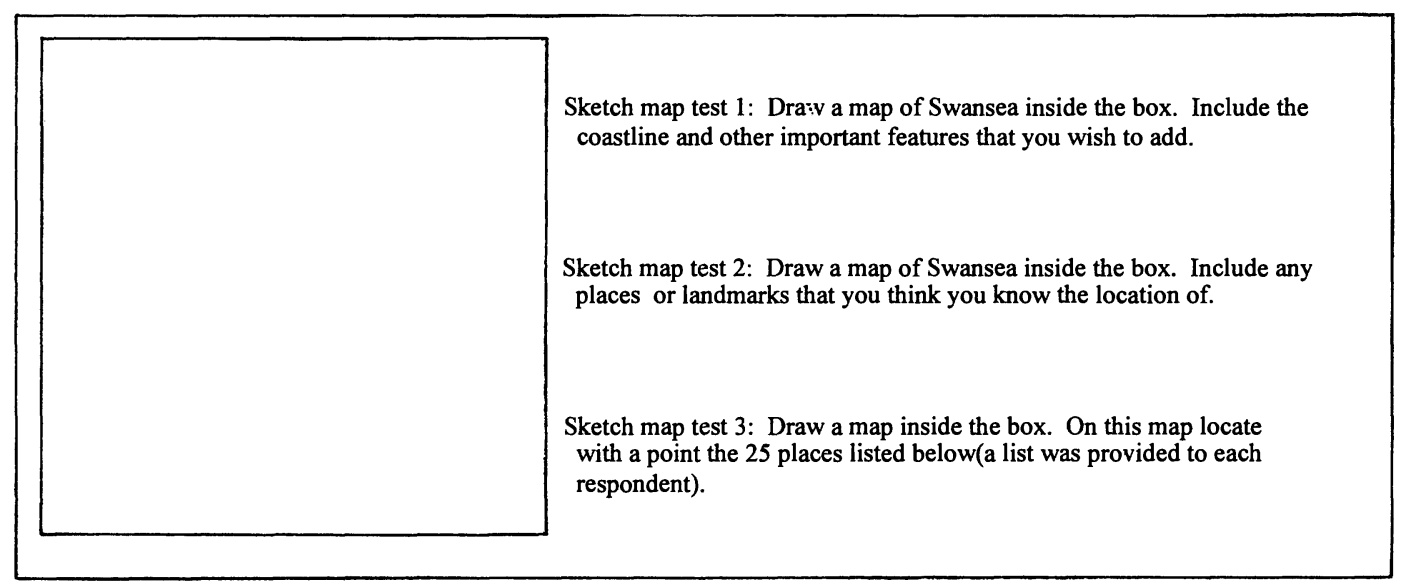

Fig. 2. The graphic tests

Harris (1981) found that amongst his respondents both men and women agreed that women have more difficulty with spatially-based tasks. Thompson et al., (1981) found that when asked 'how good is your sense of direction?' men's self-ratings were significantly higher than women's. Similarly, Lunneborg (1984) tested the metacognition of ten spatially-based activities and found that men, when comparing themselves to their peers, thought they had superior spatial abilities on nine of the tests. Lunneborg and Lunneborg (1984) found, however, that when put into practice men over-estimated their ability while females underestimated female ability. Kozolowski and Bryant (1977) did find a relationship between metacognition and performance with those with the most confidence in their ability producing significantly better results. Liben (1981) notes differences in metacognition could be the result of the fact males when faced with a difficult problem are taught to master the task, whereas females are taught to seek assistance.

Some researchers have found no differences jetween females and males performing the same spatial ability or cognitive mapping tests (Feldman and Acredolo, 1979; Gilmartin and Patton, 1984; Kirasic et al., 1984; Allen, 1988; Blades, 1990; Self et al., 1992). Indeed, Caplan et al., (1985) argue that differences between females and males are often modest in magnitude and are inconsistent from one task to another, with spatial ability being a unique construct that leads to studies with chance findings. This they contend is leading to false conclusions about differences. The evidence presented does, however, suggest females and males differ in geographic knowledge and spatial competence although the reasons for differences are unclear and could be a result of several factors.

\section{Comparing females' and males' spatial products}

Case studies, methods and analysis To test if females and males differ in their everyday geographic knowledge and spatial competence two case studies, separated by a year, were undertaken. In both studies all the 


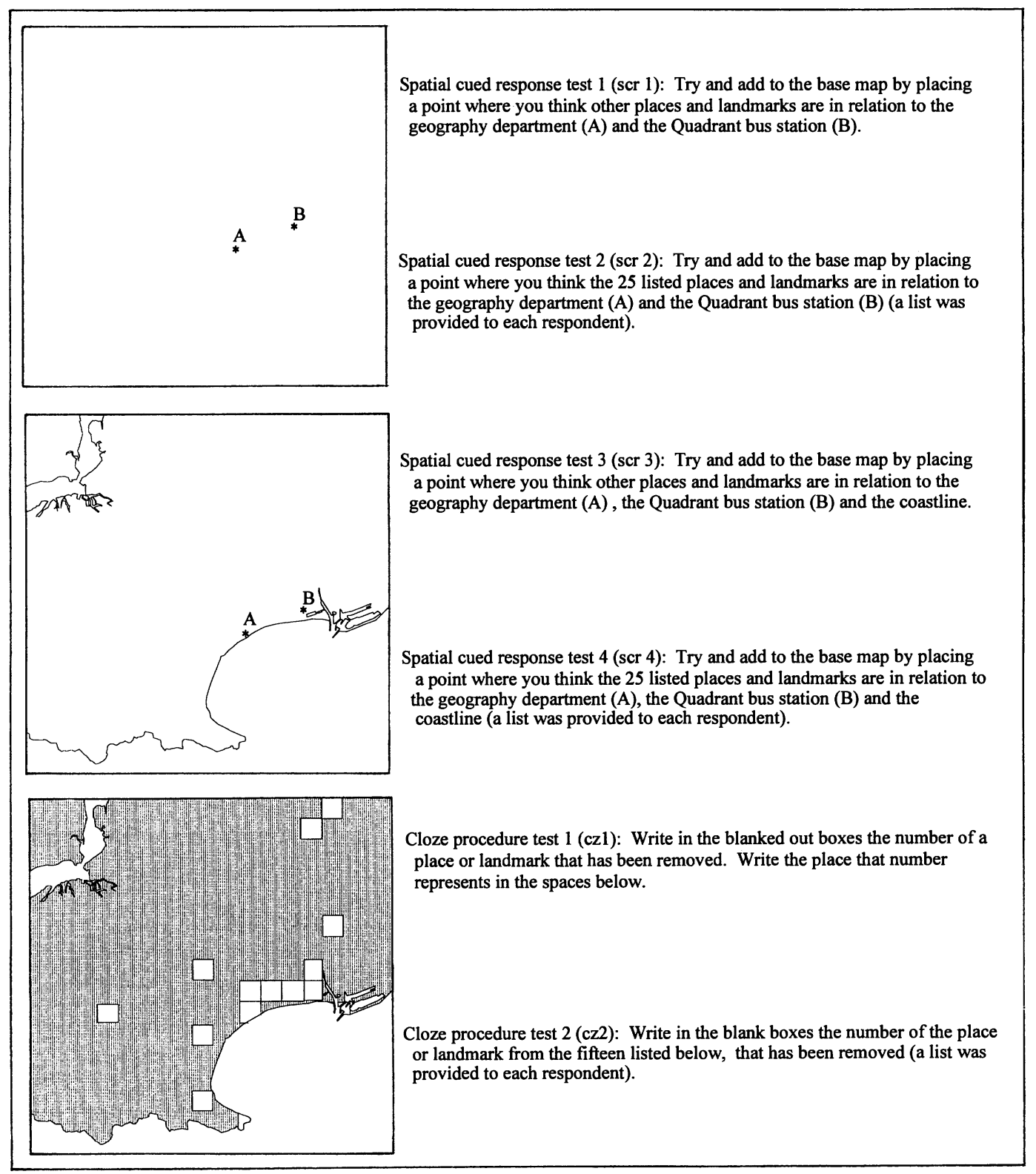

Fig. 3. The partially graphic tests

respondents were first-year undergraduates who had lived in Swansea for approximately four months. All were recruited from first-year geography practical classes and so had similar geographic training and access to maps and geographic details of the Swansea area. As part of their first year practical packs each had an Ordnance Survey Landranger (1:50000) map of the Swansea and Gower area. The practicals for the first term had provided them with map design and understanding skills and knowledge of the South
Wales area; practicals tended to be at the West Glamorgan and South Wales scale rather than the Swansea scale. All had been on a department geohistoric tour of the city and its surrounding area in the second week of arrival. It was noted that these respondents had access to information and skills not normally available to most students.

Both studies investigated the respondents' knowledge of Swansea and the surrounding area. Swansea is a coastal city composed of approximately 168000 


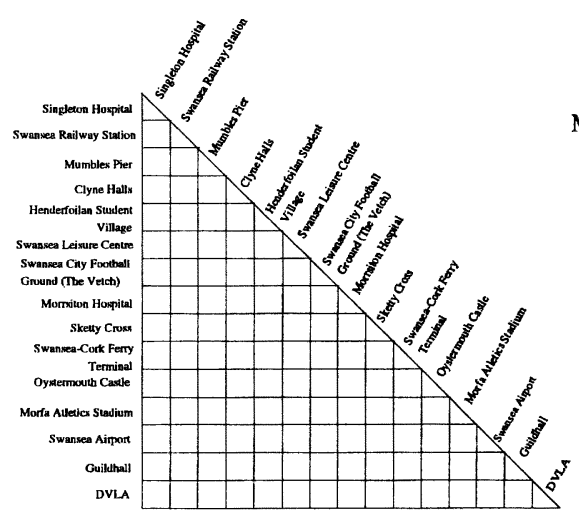

Metric MDS test: Complete the matrix, filling in the distances in miles between places listed at the ends of the rows and columns (you can use fractions of miles).

Singleton Hospital

Swansea Railway Station Mumbles Pier

1. Singleton Hospital to Swansea Railway Station.

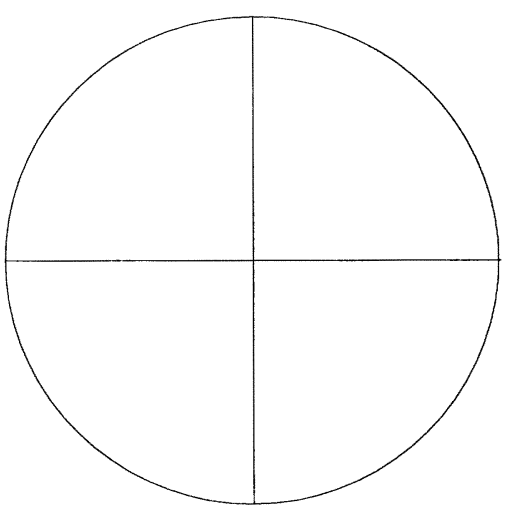

Non-metric MDS: Underline the place which is furthest away from the other two. In the example, Mumbles Pier is underlined as it is further away from the other two places.

Projective convergence test: The centre of the compass represents the first place. Mark with a line the direction to the second place. The length of the line will represent the distance from the first to the second place where the compass radius is equal to the straight line distance between Mumbles Pier and Morriston Hospital, which is 8.1 miles.

Fig. 4. The uni-to-multidimensional tests

inhabitants containing all the facilities of any large regional city. The city can be divided into four sections: a highland central section, east and west axes and a centre where these three regions meet (Fig. 1). The east axis occupies the Swansea Valley and corresponds to the River Tawe. The west axis follows the curve of the bay. Although the university and its accommodation is exclusively in the west of the city the study area covered the whole of Swansea City District area. This allowed the study of respondents' knowledge of areas that were unfamiliar or visited infrequently. By keeping the opportunities for exploration and the age of the respondents the same it is hoped that any differences can be chiefly attributed to sex, although it is appreciated that other factors, known to influence spatial behaviour such as sexuality (Adler and Brenner, 1991), might be involved.

In the first study, 177 respondents - 93 females and 84 males - were divided into six groups. Each group's respondents undertook a series of four differ- 


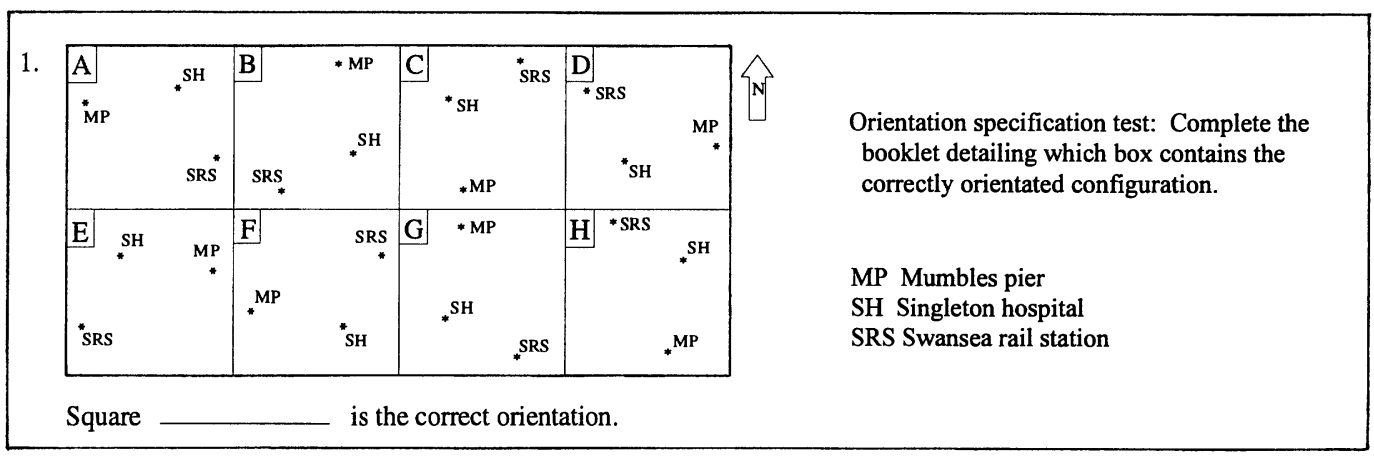

Fig. 5. The recognition test

TABLE II

Two sample $t$-tests comparing females' and males' selected bidimensional regression results variables

Test

Sketch map test 3
Spatial cued
response test 4
Metric MDS
Non-metric MDS
Projective convergence

\begin{tabular}{|c|c|c|c|}
\hline \multicolumn{2}{|c|}{$r^{2}$} & \multicolumn{2}{|c|}{ scale } \\
\hline $\mathrm{t}$ & $\mathrm{p}$ & $\mathrm{t}$ & $\mathrm{p}$ \\
\hline 0.57 & 0.58 & -0.33 & 0.98 \\
\hline-0.30 & 0.77 & -0.04 & 0.97 \\
\hline-0.70 & 0.49 & - & - \\
\hline 0.89 & 0.38 & - & - \\
\hline-0.88 & 0.39 & - & - \\
\hline
\end{tabular}

$\dagger$ significant at the $95 \%$ confidence level

ent cognitive mapping tests from a total set of 13 and their spatial products (externalized form of their knowledge e.g. a sketch map) were compared. However, because these results represent a sub-sample of a larger study designed to test the construct validity (are the tests measuring what they are supposed to?) and convergent validity (are tests that are meant to be measuring the same phenomena producing similar results?) they can only be statistically compared with confidence between eight of the tests because of low sample sizes (Table I). Visual analysis can be used to a limited degree to compare the remaining five tests, especially those involving cartographic analysis.

The 13 tests chosen for comparison were drawn from four groupings of tests identified by Kitchin (1995) and were selected to provide a diverse range of task demands. Graphic tests all involve some form of sketch mapping. Respondents undertook one of three different variations (Fig. 2). Partially graphic tests reduce the level of graphicacy needed for the respondent to complete the task. They can be divided into two categories: spatial cued response tests where the respondent is provided with a certain amount of spatial information and asked to complete the task; and cloze procedure tests where the respondent has to determine what places are located at shown sites. In total, six partially graphic tests were completed, four spatial cued response tests and two

\begin{tabular}{|c|c|c|c|c|c|}
\hline \multicolumn{2}{|c|}{ angle } & \multicolumn{2}{|c|}{$a 1$} & \multicolumn{2}{|c|}{$a 2$} \\
\hline$t$ & $\mathrm{p}$ & $t$ & $p$ & $t$ & $\mathrm{p}$ \\
\hline-1.01 & 0.32 & - & - & - & - \\
\hline 0.18 & 0.86 & 0.32 & 0.75 & 0.70 & 0.49 \\
\hline - & - & - & - & - & - \\
\hline- & - & - & - & - & - \\
\hline- & - & - & - & - & - \\
\hline
\end{tabular}

cloze procedure tests (Fig. 3). Uni-to-multidimensional tests use one-dimensional data, such as distance and direction, to construct two-dimensional spaces. They can be divided into two categories: multidimensional scaling tests use a series of algorithms to convert interval or ordinal distance data into a configuration; and projective convergence tests which use a resection technique to convert either just direction, or distance and direction, into a configuration. Respondents completed one of three unito-multidimensional tests (Fig. 4). Recognition tests provide the respondents with a representation of an environment and ask them to identify features and configurations correctly. One such test was used in the present study (Fig. 5).

Because of the diversity of the data collected a number of different methods of analysis was employed, although wherever possible consistency was attempted with bidimensional regression being most commonly used. Data from sketch map test three, all the spatial cued response tests, the metric and non-metric multidimensional scaling tests and the projective convergence test were all analysed using bidimensional regression. Bidimensional regression measures the association between configurations (Tobler, 1965) and postulates a regression-like relationship, that is basically an extension of ordinary product moment (Pearsonian) correlation and ordi- 
SEX DIFFERENCES IN GEOGRAPHICAL KNOWLEDGE AND UNDERSTANDING

281

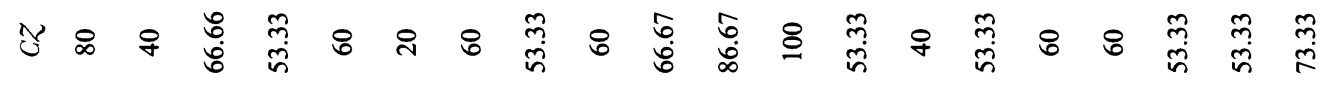

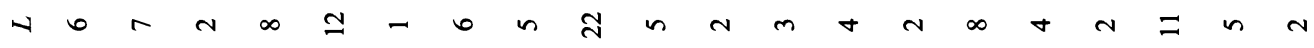

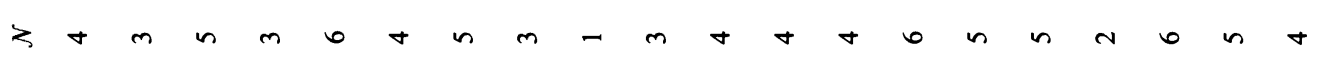

a b o h n ta d

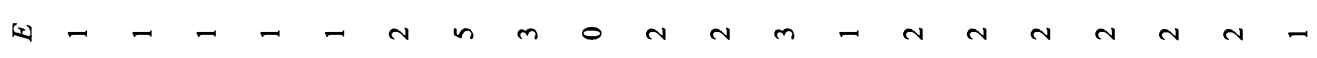

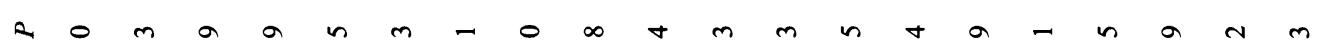

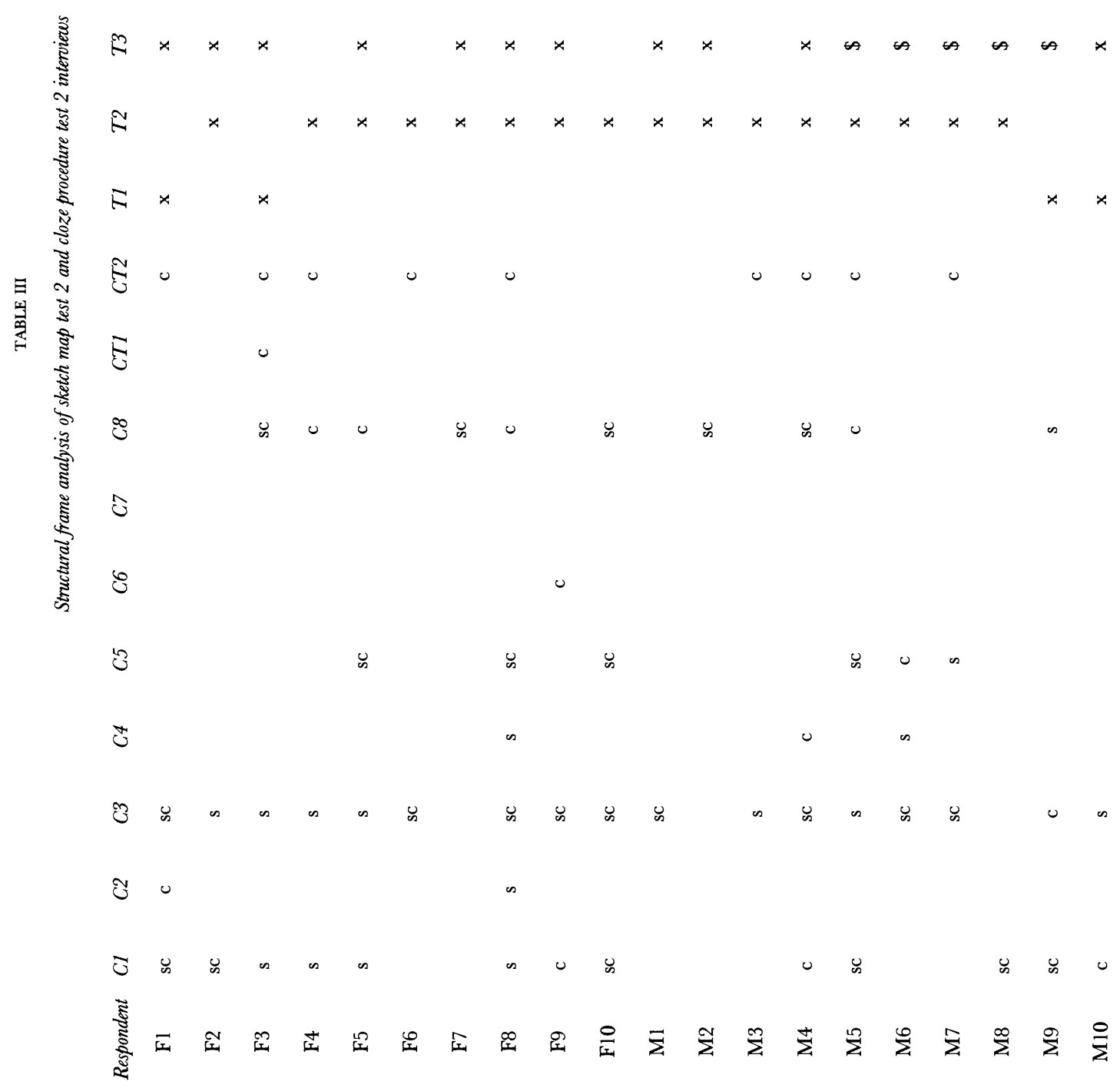




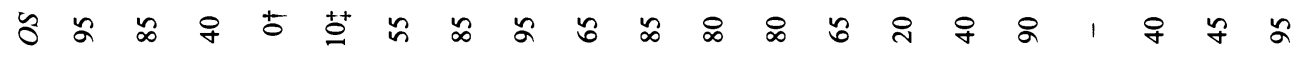

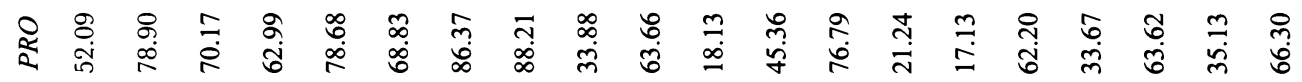
$\bar{i}$

우

R

$\infty$

F

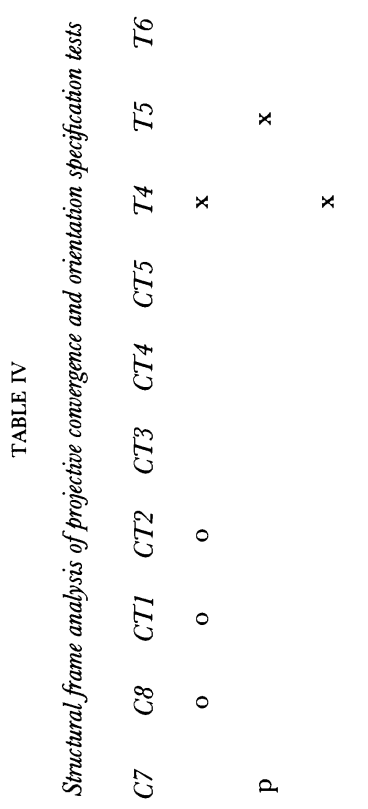

ङ

2.

3

200

2

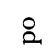

$\circ$

$心$

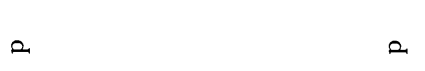

$\AA$

O a a a a \& a a a a a a

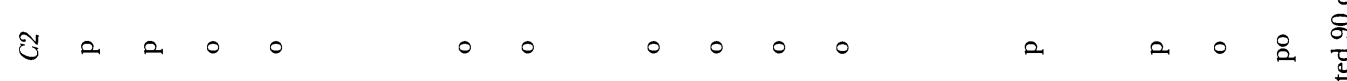

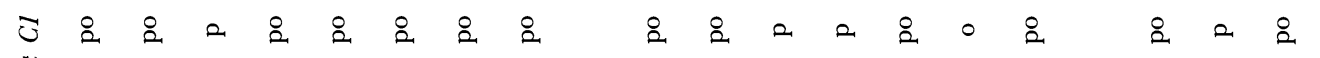

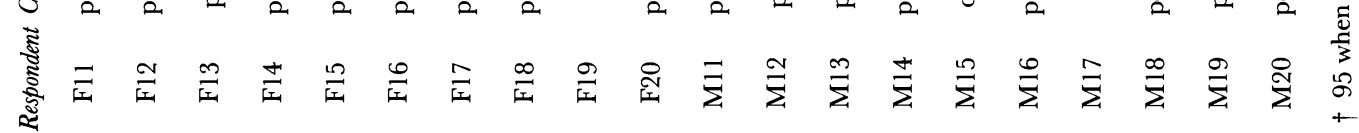


TABLE V

The strategy structural frames and the codes for Tables III/IV

$\begin{array}{ll}\text { Code } & \text { Description } \\ \text { Common } & \text { stragegies } \\ \text { C1 } & \text { Imagining or constructing various types of maps } \\ \text { C2 } & \text { Referring to the coastline } \\ \text { C3 } & \text { Imagining the route or travelling between two locations } \\ \text { C4 } & \text { Using travel time to work out the separation between locations } \\ \text { C5 } & \text { Imagining standing at a location and 'looking' in the direction of another location } \\ \text { C6 } & \text { Imagining looking down vertically or obliquely } \\ \text { G7 } & \text { Working out where places are in relation to the current location } \\ \text { C8 } & \text { Just know - propositional coding } \\ & \\ \text { Common } & \text { task strategies } \\ \text { GT1 } & \text { Elimination } \\ \text { GT2 } & \text { Logical deduction } \\ \text { GT3 } & \text { Draw a map } \\ \text { GT4 } & \text { Where the sun sets } \\ \text { CT5 } & \text { Look back at former answers }\end{array}$

Task-specific strategies

Cloze procedure

Tl Look at square, decide what is located there and check to see if on list

T2 Look at list to see if recognise any, then go to the map to see if square is free where you think it is

T3 Swap strategy

Projective convergence

T4 Work out the direction between two locations by working out the direction from each place to a third place. e.g. for A to $\mathrm{B}$ work out direction from $\mathrm{A}$ to $\mathrm{C}$, and then $\mathrm{C}$ to $\mathrm{B}$

T5 Imagine flying as a crow would between two locations

T6 Instead of working out the route from A to B, work out B to A

T7 Draw a route map across the projective convergence circle

T8 Imagining a map directly in front of yourself and lining a pencil up between the two locations and moving the pencil down across the circle

Orientation specification

T9 Work out how it should look like then scan all the squares for one that fits

T10 Work out how it should look like then work systematically through the squares until one fits, choose that and ignore the rest of the squares

T11 Draw the coastline onto the configurations
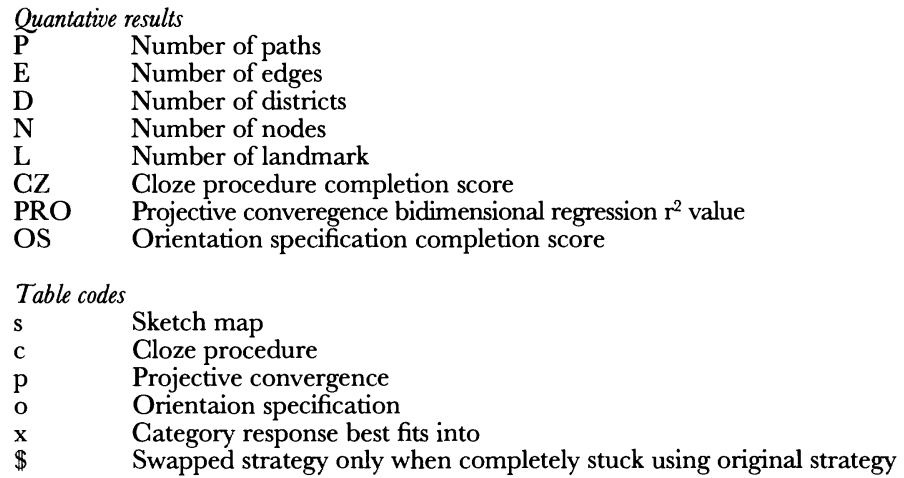

nary least squares regression procedures, between two sets of coordinates. This regression technique is sensitive to rotations, translations and changes of scale, and calculates how large these are (Tobler, 1976). A number of results variables are produced:

$\mathrm{r}^{2}$ represents the goodness-of-fit between the two sets of coordinates; scale is an index that measures the scale change needed to produce the best fit with a value less than one indicating that $u, v$ (cognitive) space needs to be contracted to fit the $\mathrm{x}, \mathrm{y}$ (reality) space, and a scale value greater than one that the $u, v$ (cognitive) space needs to be expanded;

angle is the degrees the coordinates axes must be rotated to produce the best fit, with a positive value 
indicating a counterclockwise rotation, and a negative value a clockwise rotation;

$\mathrm{a}_{1}$ is the horizontal translation needed to produce a best fit, with a positive value indicating a west-to-east shift and a negative value indicating an east-to-west shift; $a_{2}$ is the vertical translation needed to produce a best fit, with a positive value indicating a south-tonorth shift and a negative value indicating a northto-south shift (Lloyd, 1989).

Of the remaining tests, data from sketch map tests 1 and 2 were analysed using Lynch's (1960) map content classification and Pocock's (1976) map style classification. Both cloze procedure tests 1 and 2 were analysed to produce individual accuracy scores. An individual accuracy score represents how well an individual did in assigning places to the boxes. A score of 100 means all the boxes had correct locations assigned to them, and a value of 0 that all the boxes had an incorrect place assigned to them. The orientation specification data was also analysed by creating an individual accuracy score. This represented the number of configurations correctly identified. Again, the score ranges between 0 and 100 , with a value of 0 indicating that the respondent had not correctly identified any configuration and a value of 100 indicating that all the configurations had been correctly identified.

In the second study, 40 respondents - 20 females and 20 males - were divided into two groups. The first group, consisting of 10 females and 10 males, undertook the sketch map test 2 and cloze procedure test 2. The remaining respondents undertook the projective convergence test and the orientation specification test. All the respondents completed the tests using a think-aloud protocol procedure. Think-aloud protocols were developed by Newell and Simon (1972) and consist of the respondent describing their actions or thoughts while performing a task; it is literally a running commentary. By using a protocol procedure it is hoped that the knowledge and cognitive processes used in problem-solving can be discovered. The think-aloud protocols were followed by a semistructured debriefing interview which aimed to validate the protocol analysis, and to allow further discovery concerning the nature of the tests. The interviewer also gained evidence by watching reactions and gauging the extent of opinion. All respondents completed the tests whilst facing north in a room where only the front of the building opposite was visible through the window. The interviews were taped and analysed first through direct transcription and then using a series of structural frames which acted as a set of filters. The tests were all analysed using the same techniques as the first study, so that the relationship between externalized knowledge and the reported strategies of spatial thought could be examined. The debriefing interview aimed to validate the protocol analysis and also to allow further discovery concerning the nature of the tests.

Results The results from both the first and second studies indicate that there were very few differences in either geographic knowledge or in spatial ability. For example, two sample t-tests were calculated for the various bidimensional regression variables used to analyse the sketch map test 3 data. The results (Table II) indicate that there were no significant differences between females and males on any of the comparable variables. Similarly, Table II indicates that there were no differences in any of the bidimensional regression variables for spatial cued response test four or any of the uni-to-multi-dimensional tests. Likewise, the orientation specification tests produced no significant differences between males and females $(\mathrm{t}=0.32, \mathrm{p}=0.75)$. Because of small sample sizes, no statistical comparisons were made for the remaining partially graphic tests, although visual analysis revealed remarkable similarities.

Results from the second study appear to indicate that there were no differences in the type, or adoption, of strategies of spatial thought. Females and males both used the same common strategies and task-specific strategies of thought (see Kitchin and Jacobson, forthcoming), as Tables III and IV illustrate (Table V for codes). Table III, does, however, reveal that although the strategies adopted were the same, on the cloze procedure test the time of their adoption differed with males only swapping strategy once they had become completely stuck. Females, in contrast, swapped strategies much earlier. Interestingly, this difference in the use of the same strategies did not lead to significantly different results, but does suggest that males are more likely to continue using a successful strategy until it becomes redundant, whereas females explore different ways of attempting the task using the most appropriate on each individual question. In general then, females and males with similar education and time in an area, think about space in the same ways, using the same strategies.

The only difference between females and males in the first study was from sketch map test 1 where there was a significant difference in the number of elements in each of Lynch's (1960) map content category $($ chi-square $=22.74, \mathrm{p}>0.95)$. This difference was not repeated for sketch map 2 where the instructions were more specific (chi-square $=3.32$, $\mathrm{p}<0.95)$. Further analysis of the data reveals that the difference on sketch map test $l$ is caused by males making their maps more path orientated and females more node orientated. However, this difference is not apparent when comparing map styles using Pocock's (1976) classification (chi-square $=2.98, \mathrm{p}<0.95)$ where maps containing more linear features may be expected to be given a sequential 
(linear) rather than spatial (pattern) classification. It could be that the difference might be caused by females and males giving different interpretations as to which features of the spatial landscape are important, with various features holding more salience for each group. In this case, males included more linear features, predominately roads, and females more nodal features such as the university, railway station, halls of residence and shopping areas.

In the second study, females and males were found to produce differing results for both the content classification on sketch map test 2 (chi-square $=9.173$, $\mathrm{p}>0.95)$ and the $\mathrm{r}^{2}$ value on the projective convergence test $(t=2.80, p=0.013)$. The difference on the sketch map test 2 can be explained by a large anomaly caused by one respondent placing a very large number of landmarks. Once this is removed the difference disappears. The difference on the projective convergence test occurs only in an interview environment and for just this test. It may be that this group was exceptional, or that the females are better at this type of test and the situation of the interview has encouraged them to rectify any underachieving present in the non-interview situation. The small size of the sample ( 10 females, 10 males) makes it difficult to draw any definitive conclusions.

\section{Conclusions}

The evidence presented suggests that differences in cognitive map knowledge and abilities between males and females are limited when given the same geographic training and patterns of spatial behaviour. The only tests where any differences occurred involved the test where question interpretation was unconstrained (sketch map 1), the projective convergence test in the interview condition and the using of task-specific strategies on the cloze procedure test in the interview condition. However, the results have to be treated with a certain amount of caution because of the sample of respondents used in this study. Traditionally, studies comparing males and females are carried out on children whilst investigating age-related differences (for example, Matthews, 1986). The respondents taking part in this study all had approximately the same education, including specialized geographic skills, and no social or traditional female home range constraints or potential differences caused by puberty hormonal changes. The results suggest that by the age of 18 , given no age, education and social differences, males and females have equal cognitive mapping knowledge and ability. This does not mean that differences do not exist at a younger age caused by home range constraints imposed by parents and/or puberty hormone levels. It also does not mean that differences do not exist in later life caused by different courses of education leading to the development of different skills and social and cultural stereotyping. It does, however, provide evidence that any differences occurring in adulthood are likely to be socially and culturally produced, greatly influenced by genderconstricting roles. Thus differences are not hormonal or biologically based; given the same geographic training and similar patterns of spatial behaviour, females and males have equivalent geographic knowledge and spatial skills. These results conform to those found by Golledge et al., (1993) whose study compared female and male respondents who had either received formal geographic training or received no geographic training. The respondents completed a series of route learning, distance estimation and angle estimation tasks. Female geographers completed the tasks most successfully. However, female non-geographers scored the lowest marks suggesting that training and background were the factors affecting performance, not sex. Further, the results indicate that geographic education does not need to be tailored to meet any specific needs of either sex, and that females do have the same aptitude to learn and develop the spatial skills necessary for scientific and technical careers.

\section{Acknowledgements}

This work was carried out whilst the author was a teaching assistant at the University of Wales Swansea, and he would like to thank the 1993-94 and 1994-95 geography intake for undertaking the tasks for no reimbursement. The author would also like to thank the referees for their helpful and sound advice.

\section{REFERENCES}

$\rightarrow$ Adler, S. and Brenner, J. 1991 Gender and space: lesbians anc $\rightarrow$ Blades, M. 1990 The reliability of data collected from sketch gay men in the city. Int. 7. Urban Res. 16: 24-34.

$\rightarrow$ Allen, G.L. 1988 The acquisition of spatial knowledge unde $\rightarrow$ Blough, P. and Slavin, L. 1987 Reaction-time assessments of conditions of temprospatial discontinuity. Psychol. Res. 50: 183-90.

$\rightarrow$ Appleyard, D. 1970 Styles and methods of structuring a city $\rightarrow$ Bowers, C. and LaBarba, R. 1988 Sex differences in the lateriEnviron. Behav. 2: 100-17.

$\rightarrow$ Beatty, W. and Troster, A. 1987 Gender differences in geographical knowledge. Sex Roles 16: 565-89.

Bettis, N.C. 1974 An assessment of the geographical knowledge and understanding of fifth-grade students in Michigan. Doctoral dissertation. Michigan: Michigan State University.

maps. F. Environ. Psychol. 10: 327-39.

gender differences in visual - spatial performance. Perceptual and Psychophysics 1: 276-81.

zation of spatial abilities: a spatial component analysis of extreme group scores. Brain and Cognition 8: 165-77.

$\rightarrow$ Brown, M.A. and Broadway. M.J. 1981 The cognitive maps of adolescents: confusion about inter-town distance. Prof. Geogr. 33: 315-25.

$\rightarrow$ Caplan, P., MacPherson, G. and Tobin, P. 1985 Do sex differ- 
ences in spatial abilities exist: a multilevel critique with neu $\rightarrow$ Madden, J. and White, M. 1980 Spatial implications of data. Am. Psychologica 40: 786-99.

Downs, R.M. and Stea, D. 1973 Theory. In Downs, R.M. and Stea, D. (eds) Image and environment. Chicago: Aldine: 1-7.

$\rightarrow$ Feldman, A. and Acredolo, L. 1979 The effect of active vs passive exploration on memory for spatial location in chil. dren. Child Dev. 50: 698-704.

$\rightarrow$ Fengold, A. 1988 Cognitive gender differences are disappearing. Am. Psycholgica 43: 95-103.

$\rightarrow$ Gilmartin, P. and Patton, J.C. 1984 Comparing the sexes on spatial abilities: map-use skills. Ann. Ass. Am. Geogr. 74: 605-19.

Golledge, R.G., Dougherty, V.J. and Bell, S.M. 1993 Survey versus route-based wayfinding in unfamiliar environments. Paper presented at 89th Annual meeting of the Associatior $\rightarrow$ of American Geographers, 6-10th April, Atlanta, GA.

$\rightarrow$ Goodchild, B. 1974 Class differences in environmental perception: an exploratory study. Urban Stud. 11: 157-69.

$\rightarrow$ Hanson, S. and Hanson, P. 1980 Gender and urban activity patterns in Uppsala, Sweden Geogr. Rev. 70: 291-9.

$\rightarrow$ Hanson, R. and Pratt, G. 1988 Spatial dimensions of the gender division of labor in a local labor market. Urban Geogr. 9: $180-202$.

Harris, L.J. 1981 Sex related differences in spatial ability. In Liben, L.S., Patterson, A. and Newcombe, N. (eds) Spatial representation and behaviour across the life span. New York Academic Press: 83-125.

Hart, R. 1979 Children's experience of place. New York: Irvington. $\rightarrow$

$\rightarrow$ Herman, J.F. and Siegel, A.W. 1978 The development of cognitive mapping of large-scale environment. 7. Expl Child Psychol. 26: 389-406.

$\rightarrow$ Kirasic, K.C., Allen, G. and Siegel, A.W. 1984 Expression of configurational knowledge of large-scale environments student's performance of cognitive tasks. Environ. Behav. 16: $687-712$.

$\rightarrow$ Kirasic, K.C., Allen, G. and Haggerty, D. 1992 Age-relatec differences in adults macrospatial cognitive processes. Exp. Ageing Res. 18: 33-9.

Kitchin, R.M. 1995 Issues of integrity and validity in cognitive $\rightarrow$ mapping research: investigating configurational knowledge. Unpublished PhD. Swansea: University of Wales Swansea.

-, forthcoming Exploring spatial thought. Environ. Behav.

Kitchin, R.M. and Jacobson, R.D. forthcoming Techniques to collect and analyse the cognitive map knowledge of people with visual impairments or blindness: issues of validity 7. Visual Impairment and Blindness

$\rightarrow$ Kozlowski, L.T. and Bryant, K.J. 1977 Sense of direction, spatial orientation, and cognitive maps. 7. Exp. Psychol. human perception and performance 3: 590-8.

Liben, L. 1981 Spatial representation and behaviour: multiple perspectives. In Liben, L., Patterson, A.M. and Newcombe, N. (eds) Spatial representation and behaviour across the life span New York: Academic Press.

$\rightarrow$ Lloyd, R. 1989 Cognitive maps: coding and decoding information. Ann. Ass. Am. Geogr. 79: 101-24.

$\rightarrow$ Lord, F.E.A. 1941 A study of spatial orientation of children. 7. Educational Res. 34: 481-505.

$\rightarrow$ Lunneberg, C.E. and Lunneborg, P.W. 1984 Contribution of sex-differentiated experiences to spatial and mechanical reasoning abilities. Perceptual and Motor Skills 59: 107-13.

$\rightarrow$ Lunneborg, P.W. 1984 Sex differences in self-assessed everyday spatial abilities: differential practice or self-esteem? Perceptual and Motor Skills 58: 213-14.

Lynch, K. 1960 The image of the city. Mass: MIT Press. increases in the female labour force: a theoretical and empirical synthesis. Land Econ. 56: 432-46.

Matthews, M.H. 1986 Gender, home range and environmental cognition. Trans. Inst. Br. Geogr. NS12: 43-56.

McGee, M. 1979 Human spatial abilities: psychometric studies and environmental, genetic, hormonal and neurological influences. Psychol. Bull. 86: 889-918.

-, 1982 Spatial abilities: the influence of genetic factors. In Potegal, M. (ed.) Spatial abilities: development and physiological foundations. New York: Academic Press: 199-222.

McGuiness, D. and Sparks, J. 1983 Cognitive style and cognitive maps: sex differences in representation of a familiar terrain. 7. Ment. Imagery 7: 91-100.

Miller, L.K. and Santoni, V. 1986 Sex differences in spatial abilities: strategic and experiential correlates. Acta Psychologica 63: 225-35.

$\rightarrow$ Moore, G.T. 1979 Knowing about environmental knowing: the current state of theory and research on environmental cognition. Environ. Behav. 11: 33-70.

Newell, A. and Simon, H.A. 1972 Human problem solving. Englewood-Cliffs, NJ: Prentice Hall.

$\rightarrow$ Pain, R. 1991 Space, sexual violence and social control: integrating geographical and feminist analyses of women's fear of crime. Prog. Human Geogr. 15: 415-31.

Pocock, D.C.D. 1976 Some characteristics of mental maps: an empirical study. Trans. Inst. Br. Geogr. NS 1: 493-512.

Preston, V., McLafferty, S. and Hamilton, E. 1993 The impact of family status on black, white, and hispanic women's commuting. Urban Geogr. 14: 228-50.

Rose, G. 1993 Feminism and geography: the limits of geographical knowledge. Cambridge: Polity Press.

Rutherford, B. and Wekerle, G. 1988 Captive rider, captive labour: spatial constraints on women's employment. Urban Geogr. 9: 173-93.

Self, C.M., Gopal, S., Golledge, R.G. and Fenstermaker, S. 1992 Gender-related differences in spatial abilities. Prog. Human Geogr. 16: 315-42.

Self, C.M. and Golledge, R.G. 1994 Sex-related differences in spatial ability: what every geography educator should know. 7. Geogr. 93: 234-43.

$\rightarrow$ Siegel, A.W. and Schadler, M. 1977 The development of young children's spatial representations of their environment. Child Dev. 48: 388-94.

Spencer, C. and Weetman, M. 1981 The microgenesis of cognitive maps: a longitudinal study of new residents of an urban area. Trans. Inst. Br. Geogr. 6: 375-84.

Thompson, E.G., Harris, L.J. and Mann, I. 1981 Relationships among sex, measures of cognitive complexity, and performance on spatial tasks in college students. Br. F. Psychol. 72: 249-56.

Tobler, W.R. 1965 Computation of the correspondence of geographic patterns. Papers and proceedings of the Regional Science Association 15: 131-9.

-, 1976 The geometry of mental maps. In Golledge, R.G. and Rushton, G. (eds) Spatial choice and spatial behaviour. Columbus: Ohio State University Press: 69-82.

Valentine, G. 1990 Women's fear and the design of public space. Built Environ. 16: 279-87.

Witelson, S.F. and Swallow, J.A. 1988 Neuropsychological study of the development of spatial cognition. In StilesDavis, J., Kritchevsky, M. and Bellugi, U. (eds) Spatial cognition: brain bases and development. Hillsdale, NJ: Lawrence Erlbaum Associates: 373-409. 